\title{
Diferencias de género en la percepción de la ciudadanía española sobre la Ciencia de Datos ${ }^{1}$
}

\section{Gender differences regarding Spanish citizens' perception of Data Science}
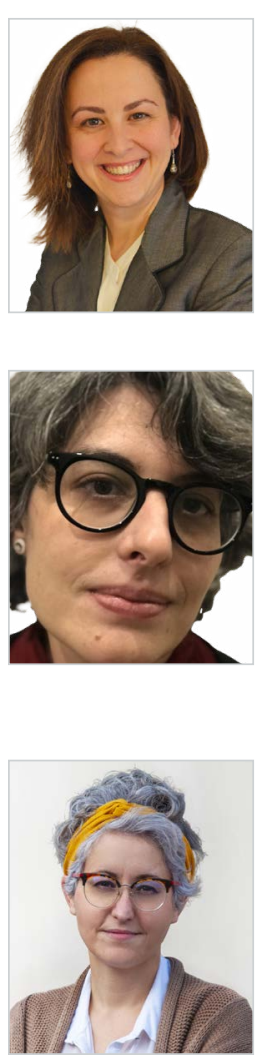

Patricia Sánchez-Holgado. Licenciada en Publicidad y Relaciones Públicas por la Universidad Complutense de Madrid. Investigadora en la Universidad de Salamanca y miembro del Observatorio de los Contenidos Audiovisuales (OCA). Actualmente es Directora de Comunicación e Innovación de la agencia de publicidad tthegap y responsable del Laboratorio de investigación. Es profesora asociada en la Facultad de Lenguas y Educación de la Universidad Nebrija de Madrid y en la Facultad de Comunicación de la Universidad Pontificia de Salamanca. Experta en Big Data y Data Science por la Universidad Pontificia de Salamanca y Máster en Estudios de la Ciencia, la Tecnología y la Innovación, con especialidad en Cultura Científica.

Universidad de Salamanca, España

patriciasanc@usal.es

ORCID: 0000-0002-6253-7087

María Marcos-Ramos. Licenciada en Comunicación Audiovisual por la Universidad del País Vasco y doctora en Comunicación Audiovisual por la Universidad de Salamanca (Premio Extraordinario). Es profesora ayudante de Comunicación Audiovisual de la Universidad de Salamanca y miembro colaborador del Observatorio de Contenidos Audiovisuales de la misma institución. Sus líneas de investigación versan sobre representación de la inmigración y del género en la ficción audiovisual, representación cinematográfica de la sociedad en el cine y análisis de la ficción audiovisual, etc. temas sobre los que ha publicado artículos en revistas y monografías científicas. Es editora de dos libros: Cine desde las dos orillas: directores españoles y brasileños (Andavira, 2018) y A ambos lados del Atlántico: películas españolas y brasileñas premiadas (Ediciones Universidad de Salamanca, 2020).

Universidad de Salamanca, España

Mariamarcos@usal.es

ORCID: 0000-0003-3764-7177

Beatriz González-de-Garay Domínguez. Profesora Contratada Doctora en Comunicación Audiovisual en la Universidad de Salamanca (España), coordinadora de la Unidad de diversidad afectivo-sexual y de identidad de género de esa Universidad y miembro del Observatorio de los Contenidos Audiovisuales, Unidad de Investigación Consolidada de Castilla y León. Licenciada con Premio Extraordinario Fin de Carrera en Comunicación Audiovisual por la Universidad Carlos III de Madrid y Doctora Europea por la Universidad Complutense. Sus principales ámbitos de investigación son los Estudios de género y diversidades sexogenéricas en la ficción televisiva, campos en los que cuenta con numerosas publicaciones académicas y proyectos de investigación.

Universidad de Salamanca, España

bgonzalezgaray@usal.es

ORCID: 0000-0002-0382-0640

1 Este trabajo forma parte del proyecto DATASCIENCE SPAIN, sobre el conocimiento y la percepción de la ciencia de los datos, el big data y la inteligencia artificial, desarrollado en la Universidad de Salamanca, por miembros del Observatorio de los Contenidos Audiovisuales. Está financiado con referencia FCT-18-13437, por la Fundación Española para la Ciencia y la Tecnología (FECYT), organismo perteneciente al Ministerio de Ciencia e Innovación de España, en la Convocatoria de ayudas para el fomento de la cultura científica, tecnológica y de la innovación.

\footnotetext{
Cómo citar este artículo:

Sánchez-Holgado, P.; Marcos Ramos, M. y González de Garay Domínguez, B. (2021). Diferencias de género en la percepción de la ciudadanía española sobre la Ciencia de Datos. Doxa Comunicación, 33, pp. 235-256.
}

https://doi.org/10.31921/doxacom.n33a1126 
Recibido: 17/03/2021 - Aceptado: 15/07/2021 - En edición: 16/09/2021 - Publicado: 01/12/2021

\section{Resumen:}

Este artículo tiene como objetivo comprobar si existen diferencias de género en el conocimiento y actitudes de la ciudadanía española hacia la ciencia de datos, además de si estas percepciones se vieron modificadas por la pandemia. Para ello se ha realizado una encuesta online con preguntas cerradas a una muestra representativa de 1105 ciudadanos y ciudadanas en dos oleadas (enero y octubre de 2020) que permitieron comparar en qué grado la pandemia por Covid-19 ejerció influencia. En los resultados se observa que en la primera oleada el conocimiento sobre Big Data e Inteligencia Artificial es moderado, siendo mayor en hombres, especialmente en Big Data; que el grado de interés disminuye en la segunda oleada en ambos géneros y apunta a varias diferencias de género en la percepción de beneficios y riesgos en sus aplicaciones: los hombres perciben más beneficios que las mujeres, mientras que las mujeres perciben más riesgos en general en todas las aplicaciones tecnológicas en la primera oleada, pero en la segunda sube su percepción de beneficios hasta casi igualar a los hombres. Se observa que en la segunda oleada los riesgos han aumentado para ambos géneros y que las diferencias entre ambos no resultan significativas.

\section{Palabras clave:}

Género; ciencia de datos; comunicación científica; Big Data; Inteligencia Artificial.
Received: 17/03/2021 - Accepted: 15/07/2021-Early access: 16/09/2021 - Published: 01/12/2021

\section{Abstract:}

The aim of this article is to determine whether there are gender differences with regard to the knowledge and attitudes of Spanish citizens toward data science, and whether those perceptions have been altered by the pandemic. For this purpose, an online survey with closed questions was conducted with a representative sample of 1,105 male and female citizens in two phases (January and October of 2020), in order to compare the degree to which the Covid-19 pandemic has influenced such perceptions. The results show that knowledge regarding Big Data and Artificial Intelligence is modest, being higher among men, especially in relation to Big Data. Moreover, the level of interest decreased in the second phase in both genders, which points to several gender differences with regard to the perception of benefits and risks of their application, such as the following: men perceived more benefits than women, while women generally perceived more risks with all technological applications in the first phase, yet in the second phase their perception of benefits rose to a level nearly equal to that of men. It has also been observed that in the second phase the perception of risk increased for both genders, and that the differences between the two are not significant.

\section{Keywords:}

Gender; data science; science communication; Big Data; Artificial Intelligence.

\section{Introducción}

Existe un interés ciudadano amplio y creciente sobre los avances científicos que, en la actualidad, entronca directamente con la pandemia del Covid-19, pero cuya trayectoria reciente proviene de la atención que los medios de comunicación prestan a temas relacionados con las nuevas tecnologías y especialmente con todo lo relacionado con el Big Data o la Inteligencia Artificial. No obstante, se trata del tradicional econiano dilema entre apocalípticos, que consideran la tecnología como una amenaza existencial para la humanidad si las máquinas se vuelven más inteligentes que los humanos, e integrados, que perciben el avance de la tecnología como sinónimo de progreso y la innovación.

Se realiza en este artículo un análisis transversal con perspectiva de género sobre los conocimientos y las actitudes de la ciudadanía española hacia la ciencia de datos. Se comprueba si existen diferencias entre hombres y mujeres en la percepción de la ciencia de datos, el Big Data y la Inteligencia Artificial en cuanto a familiarización e interés y beneficios y riesgos. Para ello se observan los resultados de una encuesta obtenidos en dos momentos temporales diferentes. La primera oleada de la encuesta se desarrolló en enero de 2020 y la segunda oleada de la encuesta se pudo llevar a cabo en octubre de 2020, estableciendo además 
una comparativa entre ambas en las variables seleccionadas. La pandemia por Covid19 y el confinamiento en España sucedió entre ambas oleadas, por lo que es de interés observar si hubo cambios en la percepción estudiada antes y después.

El sesgo de género en la Inteligencia Artificial y el Big Data ha sido ampliamente señalado ya que, como señala Leavy (2018) los desarrolladores de estas tecnologías son en su abrumadora mayoría hombres. Desde aplicaciones de reconocimiento de voz hasta los motores de búsqueda, la Inteligencia Artificial y los algoritmos basados en Big Data pueden reproducir los patrones de discriminación existentes (Howard, 2018). Se trata en este estudio de medir la percepción de la ciudadanía hacia estas realidades en el contexto español para determinar si existen diferencias entre hombres y mujeres.

\section{Estado de la cuestión}

La sociedad actual se caracteriza por la transmisión del conocimiento a través de múltiples canales, siendo los medios de comunicación de masas una de las principales fuentes informativas, con lo que vivimos una era de globalización. Durante la pandemia se ha observado que la televisión ha sido el principal medio informativo, seguido de cerca por los medios digitales (Montaña Blasco et al., 2020). La comunicación de la ciencia ha sido frecuentemente analizada en el contexto europeo e internacional (Miller, 2004; Miller, 2001; European Commission, 2014, 2017a, 2017b, 2020). La información y los datos constituyen un nuevo y valioso mercado, mientras que el importante avance tecnológico que vivimos es el que construye y sostiene la sociedad del conocimiento (Palomares Ruiz, 2004). Sin embargo, en base a esto, la ciencia desempeña un papel fundamental en el desarrollo de la sociedad y hay que realizar un esfuerzo para que su difusión llegue a todos los públicos y con ello incrementar el conocimiento científico.

La comunicación científica responde a una función informativa, de creación de cultura y de reconocimiento y apoyo para los investigadores y sus trabajos, dotándoles de alto valor social (Pearson, 2001). La comprensión pública de la ciencia requiere que se impliquen todos los actores del sector, por lo que se apela a la movilización de científicos y de recursos capaces de involucrar al público, preparar el contexto y desarrollar acciones que obtengan resultados. Por parte del público es necesario tener una voluntad de participación para poder establecer un diálogo entre los avances científico tecnológicos y sus públicos (Bauer y Jensen, 2011). Los estudios de conocimiento y percepción social de la ciencia y la tecnología aportan información sobre cómo llegar al público y cuál es su punto de partida. Nos permiten conocer el grado de conocimiento que los ciudadanos presentan, cómo se entienden los riesgos y beneficios e identificar cuestiones sociales, culturales o políticas en la opinión pública que pueden afectar a las decisiones gubernamentales que se adoptan sobre este tema. Estos estudios también facilitan el conocimiento entre los diversos agentes sociales, como los medios de comunicación, el sector investigador, la comunidad educativa o el sector privado, entre otros), de manera que conectan a científicos, comunicadores y responsables de política científica (Felt, 2007).

En la sociedad no hay un conocimiento extendido sobre los conceptos básicos de la ciencia de datos, por ser una disciplina novedosa y a la vez compleja.

La ciencia de datos es una disciplina contemporánea que combina enfoques de informática y matemáticas para obtener un conocimiento significativo de los datos. En ella se incluyen parcialmente las técnicas de Big Data y de Inteligencia Artificial. 
El Big Data es un campo que incluye el tratamiento de conjuntos de datos que tienen gran volumen, son complejos y crecen a gran velocidad, por lo que no pueden capturarse, procesarse o analizarse con técnicas convencionales (Mayer-Schönberger \& Cukier, 2013).

La Inteligencia Artificial es una combinación de técnicas que estudia cómo las máquinas pueden resolver problemas complejos razonando y eligiendo las mejores opciones y, aunque es más amplia, se incluye en parte en la disciplina de la ciencia de datos. Según la Unión Europea, la inteligencia artificial es la habilidad de una máquina de presentar las mismas capacidades que los seres humanos, como el razonamiento, el aprendizaje, la creatividad y la capacidad de planificación (Samoili et al., 2020).

Sin embargo, se observan actitudes negativas hacia el avance de estas técnicas y su aplicación en la vida diaria. En el ámbito nacional, la Fundación Española para la Ciencia y la Tecnología (FECYT) publica bianualmente desde 2002 el estudio "Percepción social de la Ciencia y la Tecnología en España" que permite saber, de manera longitudinal, las opiniones de la ciudadanía sobre estos temas. En 2018, la encuesta introduce por primera vez la percepción de riesgos sobre la Inteligencia Artificial, destacando que un 38,4\% aprecia bastante o mucho riesgo, y la mecanización o robotización del trabajo, aspecto en el que un 45,9\% de los encuestados considera que hay bastante o mucho riesgo (FECYT, 2018). En el estudio actualizado de 2020 los datos varían ligeramente, puesto que descienden a un 34,3\% las personas que aprecian bastante o mucho riesgo, y en cuanto a la mecanización o robotización del trabajo aumenta a un 48 \% de los encuestados que consideran que hay bastante o mucho riesgo (FECYT, 2021).

En el ámbito europeo los estudios también apuntan a cierto escepticismo en torno a los descubrimientos tecnológicos, especialmente en relación a que podrían destruir empleos o poner en riesgo nuestra privacidad (European Commission, 2017a). En una aplicación práctica, estudios previos han analizado las opiniones sobre el avance de la ciencia de datos en la profesión periodística, estudiando varios puntos de vista (Calvo-Rubio et al., 2020), o la percepción de los periodistas ante la propia comunicación de la ciencia de datos (Sánchez-Holgado et al., 2021).

En este contexto, la ciencia de datos es uno de los campos más punteros actualmente, pero también demuestra ser de los más controvertidos, por lo que su comunicación presenta retos añadidos a la mayoría de campos de la comunicación científica, con una carencia importante en la formación de cultura científica.

El enfoque de género en los estudios de percepción social de la ciencia ha sido estudiado previamente en múltiples estudios, como en el de Hayes y Tariz (2000), que revisaba si las mujeres en los Estados Unidos, Canadá, Gran Bretaña y Nueva Zelanda tenían menos conocimiento y actitudes menos favorables hacia la ciencia que los hombres, concluyendo que no había diferencias por una cuestión de género sino por causas socioeconómicas, formativas y culturales, debido al origen social, salvo en el caso de Estados Unidos, donde las diferencias se debían a la formación en conocimientos científicos primordialmente. También se relaciona directamente con el conocido como "efecto del hombre blanco", que apunta a que los hombres blancos perciben los riesgos de los peligros para la salud y la tecnología como bajos en comparación con las mujeres blancas y las personas de color. Las percepciones de bajo riesgo de los hombres blancos se asocian con visiones del mundo individualistas y jerarquistas en oposición a una visión del mundo igualitaria. En esta línea, Finucane et al. (2000) realizaron un estudio nacional para medir las percepciones de los riesgos para la salud ambiental atendiendo a cuestiones de género y raza, y los resultados mostraron que las mujeres percibían los riesgos mucho más elevados que los hombres (Finucane et al., 2000). 
Berman y Bourne (2015) marcan como prioridad la diversidad de género en la ciencia de datos por su importancia estratégica en las sociedades de la información y la comunicación, así como por la tradicional brecha de género en las STEM (ciencias, tecnología, ingeniería y matemáticas). La evidencia científica demuestra que "incorporar el análisis sexogenérico a la investigación puede mejorar la reproductibilidad y la eficiencia experimental, ayudar a reducir sesgos, permitir la igualdad social en los resultados científicos y fomentar oportunidades para el descubrimiento y la innovación" (Tannenbaum et al., 2019).

Si los grandes datos están sesgados, se transmite al análisis que se realiza y en última instancia al conocimiento. Por ello, ya hay esfuerzos por suprimir dicho sesgo, como la herramienta propuesta por Díaz Martínez et al. (2020) que realiza su filtrado en español y a su vez utiliza el Big Data como objeto y como recurso. No obstante, mientras que el sexo como variable biológica en la ciencia y la ingeniería ha sido tradicionalmente estudiado, el género como variable cultural es complejo y multidimensional y "las aplicaciones en campos técnicos a menudo requieren la colaboración con científicos sociales para comprender los aspectos relevantes del género para proyectos específicos" (Tannenbaum et al., 2019). En este sentido los mismos autores destacan en la revista Nature que los "desafíos metodológicos incluyen ir más allá de lo binario, femenino y masculino, mujeres y hombres, tanto en el análisis del sexo como del género". Autores como D'Ignazio y Klein (2020) resumen la importancia de la perspectiva de género en la ciencia de datos en su libro Data Feminism explicando que el feminismo interseccional examina la inequidad de poder y que en el mundo contemporáneo los datos son poder. Algunos observadores críticos alertan sobre la producción mecánica de prejuicios de raza o género, pero la naturaleza distante y multidimensional de las correlaciones del aprendizaje automático puede significar que formas más sutiles de discriminación pasen desapercibidas (McQuillan, 2018). En este sentido, Jurgenson (2014) fija como mayor problema de lo que denomina la ideología del Big Data el hecho de que los investigadores cuyo trabajo se mueve alrededor de la raza, el género y la sexualidad en la cultura se niegan a reconocer cómo su punto de vista social específico influye en todo su proceso de investigación.

El estudio de las posibles diferencias de género en la percepción social de la ciencia de datos en dos momentos temporales que representan la situación pre-confinamiento y post-confinamiento por Covid-19 añade coherencia y valor al estudio porque permite valorar el impacto que unas circunstancias extraordinarias ha tenido en la percepción de las tecnologías que utilizan Big Data e Inteligencia Artificial. En la mayoría de los sectores se han evaluado o se están evaluando los impactos que el confinamiento ha tenido en las personas, por lo que aplicarlo en este momento es relevante para los resultados de esta investigación.

En el caso de las mujeres se ha puesto de manifiesto que la responsabilidad de los cuidados familiares ha afectado directamente a su actitud, percepción y salud, y se han estudiado los efectos del Covid-19 sobre ellas desde la Organización de las Naciones Unidas (ONU Mujeres, 2021). Conocer la perspectiva de género se muestra esencial para dar respuesta a la pandemia y a sus efectos según el informe publicado por el Instituto de las Mujeres (Instituto de las Mujeres y para la Igualdad de Oportunidades, 2020) y la participación igualitaria de las mujeres en las decisiones son elementos centrales para la recuperación de la crisis según la Organización de los Estados Americanos (OEA) (Comisión Interamericana de Mujeres, 2020).

Por tanto, el enfoque de género resulta relevante en los conocimientos y en la percepción de beneficios y riesgos sobre la ciencia de datos, el Big Data y la Inteligencia Artificial y este es el vacío que pretende cubrir esta investigación. 


\section{Objetivos}

El objetivo principal de este artículo es poner de relieve las conexiones estructurales entre la percepción de la comunicación de la ciencia de datos y el género.

En base a ello, y a partir de la revisión literaria realizada, se plantean las siguientes preguntas de investigación (PI):

PI1: ¿Existen diferencias de género en el grado de conocimiento e interés de la ciencia de datos, Big Data e Inteligencia Artificial en España?

PIla: ¿Se han producido cambios entre el antes y el después del confinamiento por Covid-19?

PI2: ¿Existen diferencias de género en la percepción de beneficios y riesgos de la ciencia de datos, Big Data e Inteligencia Artificial en España?

PI2a: ¿Se han producido cambios entre el antes y el después del confinamiento por Covid-19?

\section{Metodología}

\subsection{Muestra y procedimiento}

Para abordar este trabajo se analizaron los resultados de dos oleadas de una encuesta realizada a una muestra representativa de la sociedad en España en el marco del proyecto Data Science Spain. Para ello, se diseñó y validó un cuestionario con preguntas cerradas. La distribución se realizó online en ambas oleadas a través de la plataforma Qualtrics, solicitando el servicio de muestreo a la empres para disponer de una muestra aleatorizada que garantizara una distribución adecuada y representativa de los encuestados, estratificada por género, edad y comunidad autónoma, tanto en la primera oleada como en la segunda.

La primera oleada se distribuyó en enero de 2020, entre los días 20 y 24, sobre una muestra de 684 personas, mientras que la segunda oleada se realizó en octubre de 2020, entre los días 7 y 12, a 421 personas. Así la muestra total es amplia pues consta de 1105 encuestados. Además, en los casi nueve meses de diferencia entre ambas, la pandemia por Covid-19 y el confinamiento que se decretó en España marcan de forma importante el espacio entre una y otra, por lo que debe ser analizada en qué medida esta circunstancia influyó en los encuestados.

Para poder realizar una comparativa longitudinal entre las dos oleadas, este estudio se centra en 4 bloques específicos que fueron replicados en ambas: la familiarización, el interés, los beneficios y los riesgos.

\subsection{Medidas}

Para medir el grado de conocimiento y dar respuesta a la primera pregunta de investigación, se utilizó una escala de tipo Likert compuesta por cinco puntos con las siguientes variables:

- Familiarización con el concepto Big Data y con el concepto de Inteligencia Artificial: escala de 1 (nada familiarizado, no conozco nada del tema) a 5 (muy familiarizado, comprendo su uso y lo utilizo), que mide hasta qué punto los encuestados conocen la materia y la utilizan. 
- Interés en el Big Data y en la Inteligencia Artificial, su conocimiento o su uso: escala de 1 (nada interesado) a 5 (muy interesado), que mide el grado de interés de los ciudadanos por la materia.

Para medir la percepción de beneficios y riesgos y dar respuesta a la segunda pregunta de investigación planteada se utilizó el mismo tipo de medidas con las siguientes variables:

- Beneficios de aplicaciones del Big Data y la Inteligencia Artificial: de 1 (ningún beneficio) a 5 (muchos beneficios) para medir la cantidad de beneficio percibido en cada una de las aplicaciones seleccionadas.

- Riesgos de las aplicaciones del Big Data y la Inteligencia Artificial: de 1 (ningún riesgo) a 5 (muchos riesgos), que mide la cantidad de riesgos percibidos en cada una de las aplicaciones seleccionadas.

\subsection{Análisis}

Las respuestas obtenidas fueron anonimizadas y se realizó un análisis descriptivo (de frecuencias y medias). Se aplicaron pruebas de estadística inferencial: pruebas $\mathrm{T}$ de Student para muestras independientes para investigar si existen diferencias en función del género, que se realizaron en el paquete SPSS de IBM, versión 25. Por último se calculó el valor de la d de Cohen utilizando las medias y las desviaciones estándar de los grupos.

\section{Resultados}

\subsection{Características sociodemográficas}

De las 684 personas que componen la muestra, 375 fueron mujeres y 306 hombres (Tabla 1).

Tabla 1. Distribución de la muestra por género

\begin{tabular}{|c|c|}
\hline Género & \% de la muestra \\
\hline Mujeres & $54.82 \%$ \\
\hline Hombres & $44.74 \%$ \\
\hline Otro & $0.44 \%$ \\
\hline
\end{tabular}

Fuente: elaboración propia

En cuanto a la distribución por edad la opción predominante se sitúa en la franja de edad 35 a 49 años y la de menor cobertura la de 18 a 24 años (Tabla 2). 
Tabla 2. Distribución de la muestra por edad

\begin{tabular}{|l|c|}
\hline Edad & \% de la muestra \\
\hline 18 a 24 años & $8.19 \%$ \\
\hline 25 a 34 años & $25.00 \%$ \\
\hline 35 a 49 años & $30.26 \%$ \\
\hline 50 a 64 años & $19.30 \%$ \\
\hline Más de 65 años & $17.25 \%$ \\
\hline
\end{tabular}

Fuente: elaboración propia

Se comprobó que la representatividad de la muestra estuviese equilibrada con la población de tal manera que el $62,87 \%$ de los encuestados residía en la Comunidad de Madrid, Cataluña, Andalucía y Comunidad Valenciana (Tabla 3). Estos datos están en concordancia con el número de habitantes de la población en la que residía los encuestados, pues el 24,7\% reside en una población de más de 500.000 habitantes y el 24,6\% en poblaciones de 100.001 a 500.000 habitantes. En poblaciones de entre 10.000 y 100.000 habitantes reside un $36,4 \%$ de los encuestados y un $12,6 \%$ en núcleos de menos de 10.000 habitantes.

Tabla 3. Distribución geográfica de la muestra

\begin{tabular}{lc}
\hline Comunidad Autónoma (C.A) & \% de la muestra \\
\hline Andalucía & $14.62 \%$ \\
\hline Aragón & $3.07 \%$ \\
\hline Cantabria & $1.75 \%$ \\
\hline Castilla La Mancha & $2.49 \%$ \\
\hline Castilla y León & $5.70 \%$ \\
\hline Cataluña & $15.06 \%$ \\
\hline Ceuta & $0.15 \%$ \\
\hline Comunidad de Madrid & $20.91 \%$ \\
\hline Comunidad Foral de Navarra & $0.58 \%$ \\
\hline Comunidad Valenciana & $12.28 \%$ \\
\hline Extremadura & $2.49 \%$ \\
\hline Galicia & $4.97 \%$ \\
\hline Islas Baleares & $1.17 \%$ \\
\hline
\end{tabular}




\begin{tabular}{ll}
\hline Islas Canarias & $2.78 \%$ \\
\hline La Rioja & $0.88 \%$ \\
\hline Melilla & $0.15 \%$ \\
\hline País Vasco & $4.97 \%$ \\
\hline Principado de Asturias & $3.22 \%$ \\
\hline Región de Murcia & $2.78 \%$ \\
\hline
\end{tabular}

Fuente: elaboración propia

En cuanto al nivel de estudios casi la mitad de los encuestados (48,89\%) tienen estudios superiores por lo que son gente que, a priori, han tenido contacto con la ciencia (Tabla 4).

Tabla 4. Distribución de la muestra por nivel de estudios

\begin{tabular}{|l|c|}
\hline Nivel de estudios & \% de la muestra \\
\hline Sin estudios & $0.44 \%$ \\
\hline Primaria o equivalente & $2.63 \%$ \\
\hline Educación Secundaria Obligatoria o equivalente & $8.19 \%$ \\
\hline Bachillerato & $16.81 \%$ \\
\hline Estudios de Ciclos Formativos Profesionales o equivalente (FP) & $21.93 \%$ \\
\hline Enseñanzas superiores universitarias, máster o postgrado & $39.78 \%$ \\
\hline Estudios de Tercer ciclo (Doctorados) & $10.09 \%$ \\
\hline
\end{tabular}

Fuente: elaboración propia

\subsection{Diferencias de género en el grado de familiarización e interés de la ciencia de datos, Big Data e Inteligencia Artificial en España}

Con respecto a la primera pregunta planteada (PI1), atendiendo al grado de familiarización con el Big Data o la Inteligencia Artificial, si observamos la primera oleada de enero 2020, los datos que estamos analizando mostraron valores homogéneos entre hombres y mujeres. Así, se pudo apreciar únicamente que los hombres están tendencialmente más familiarizados con el concepto de Big Data que las mujeres. En el caso de la Inteligencia Artificial no hay apenas diferencia (Tabla 5).

Si observamos la segunda oleada de octubre 2020, los valores obtenidos muestran una diferencia significativa entre hombres y mujeres, indicando que los hombres están más familiarizados con el Big Data y con la Inteligencia Artificial que las mujeres (Tabla 6).

Se puede ver que en la segunda oleada la familiarización con el Big Data y la Inteligencia Artificial es significativa, mientras que en la primera no sucedía esto, con lo que en octubre aumentan las diferencias de género en este concepto. 
Atendiendo al grado de interés y para completar la respuesta a la PI1, observamos que la primera oleada de enero 2020 muestra valores homogéneos entre hombres y mujeres en cuanto al interés hacia el Big Data y hacia la Inteligencia Artificial Data (Tabla 5).

Observando la segunda oleada de octubre 2020, los hombres están más interesados en el Big Data y en la Inteligencia Artificial que las mujeres, pero los datos no son significativos (Tabla 6).

Tabla 5 . Familiarización e interés hacia el Big Data y la Inteligencia Artificial en la primera oleada de enero 2020

\section{PRIMERA OLEADA ENERO 2020}

\begin{tabular}{|c|c|c|c|c|c|c|c|c|c|}
\hline & $\begin{array}{c}\mathbf{N} \\
\text { Hom }\end{array}$ & $\begin{array}{c}\text { M } \\
\text { Hom }\end{array}$ & $\begin{array}{c}\text { DT } \\
\text { Hom }\end{array}$ & $\begin{array}{c}\mathbf{N} \\
\mathbf{M u j}\end{array}$ & $\begin{array}{c}\text { M } \\
\text { Muj }\end{array}$ & $\begin{array}{c}\text { DT } \\
\text { Muj }\end{array}$ & t (679) & $\mathbf{p}$ & d \\
\hline \multicolumn{10}{|l|}{ Familiarización } \\
\hline Big Data & 306 & 2.97 & 1.432 & 375 & 2.77 & 1.449 & 1.819 & 0.069 & 0.14 \\
\hline Inteligencia Artificial & 306 & 3.48 & 1.244 & 375 & 3.41 & 1.300 & 0.731 & 0.465 & 0.05 \\
\hline \multicolumn{10}{|l|}{ Interés } \\
\hline Big Data & 306 & 3.64 & 1.265 & 375 & 3.64 & 1.230 & 0.012 & 0.991 & 0.00 \\
\hline Inteligencia Artificial & 306 & 3.91 & 1.196 & 375 & 3.89 & 1.212 & 0.186 & 0.853 & 0.02 \\
\hline
\end{tabular}

Tabla 6. Familiarización e interés hacia el Big Data y la Inteligencia Artificial en la segunda oleada de octubre 2020

\section{SEGUNDA OLEADA OCTUBRE 2020}

\begin{tabular}{|c|c|c|c|c|c|c|c|c|c|}
\hline & $\begin{array}{c}\mathbf{N} \\
\text { Hom }\end{array}$ & $\begin{array}{c}\text { M } \\
\text { Hom }\end{array}$ & $\begin{array}{c}\text { DT } \\
\text { Hom }\end{array}$ & $\begin{array}{c}\text { N } \\
\text { Muj }\end{array}$ & $\begin{array}{c}\text { M } \\
\text { Muj }\end{array}$ & $\begin{array}{c}\text { DT } \\
\text { Muj }\end{array}$ & $t(417)$ & $\mathbf{p}$ & d \\
\hline \multicolumn{10}{|l|}{ Familiarización } \\
\hline Big Data* & 208 & 3.00 & 1.436 & 211 & 2.66 & 1.532 & 2.352 & $<0.05$ & 0.23 \\
\hline Inteligencia Artificial* & 208 & 3.45 & 1.281 & 211 & 3.16 & 1.280 & 2.363 & $<0.05$ & 0.23 \\
\hline \multicolumn{10}{|l|}{ Interés } \\
\hline Big Data & 208 & 3.36 & 1.266 & 211 & 3.27 & 1.317 & 0.641 & 0.522 & 0.07 \\
\hline Inteligencia Artificial & 208 & 3.57 & 1.249 & 211 & 2.49 & 1.197 & 0.662 & 0.508 & 0.06 \\
\hline
\end{tabular}

Con respecto a la PIla en la que se planteaba si se habían producido cambios entre los dos períodos, lo que se observa es que en la segunda oleada de octubre descienden tanto el nivel de familiarización como de interés en hombres y en mujeres. 
En el caso de la familiarización, las mujeres están menos familiarizadas pero en octubre baja su nivel más que en el caso de los hombres, tanto en Big Data como en Inteligencia Artificial, siendo significativas las diferencias de género entre hombres y mujeres en la segunda oleada. En el caso de los hombres incluso en octubre elevan un poco su nivel de familiarización con el Big Data, resultando una diferencia significativa con las mujeres y con el tamaño de efecto elevado. En Inteligencia Artificial, a pesar de que los hombres también descienden un poco siguen presentando una diferencia significativa con las mujeres con un tamaño de efecto elevado.

En el caso del interés no hay variaciones significativas entre hombres y mujeres, mientras que de la primera a la segunda oleada desciende el nivel de interés tanto en Big Data como en Inteligencia Artificial (Tabla 7).

Tabla 7. Comparativa por género de la primera oleada en Enero y la segunda oleada en Octubre

\begin{tabular}{|c|c|c|c|c|c|c|c|c|c|c|c|}
\hline \multicolumn{2}{|c|}{ FAMILIARIZACIÓN } & $\begin{array}{c}\mathbf{N} \\
\text { Hom }\end{array}$ & $\begin{array}{c}\text { M } \\
\text { Hom }\end{array}$ & $\begin{array}{c}\text { DT } \\
\text { Hom }\end{array}$ & $\begin{array}{c}\mathbf{N} \\
\text { Muj }\end{array}$ & $\underset{\text { Muj }}{\text { M }}$ & $\begin{array}{c}\text { DT } \\
\text { Muj }\end{array}$ & gl & $\mathbf{t}$ & $\mathbf{p}$ & d \\
\hline ENERO & BD & 306 & 2.97 & 1.43 & 375 & 2.77 & 1.45 & 679 & 1.819 & 0.069 & 0.14 \\
\hline OCT & BD* $^{*}$ & 208 & 3.00 & 1.43 & 211 & 2.66 & 1.53 & 417 & 2.352 & $<0.05$ & 0.23 \\
\hline ENERO & IA & 306 & 3.48 & 1.24 & 375 & 3.41 & 1.30 & 679 & 0.731 & 0.465 & 0.05 \\
\hline OCT & $\mathbf{I A}^{*}$ & 208 & 3.45 & 1.28 & 211 & 3.16 & 1.28 & 417 & 2.363 & $<0.05$ & 0.23 \\
\hline INTERÉS & & $\begin{array}{c}\mathbf{N} \\
\text { Hom }\end{array}$ & $\begin{array}{c}\text { M } \\
\text { Hom }\end{array}$ & $\begin{array}{c}\text { DT } \\
\text { Hom }\end{array}$ & $\begin{array}{c}\text { N } \\
\text { Muj }\end{array}$ & $\begin{array}{c}\text { M } \\
\text { Muj }\end{array}$ & $\begin{array}{c}\text { DT } \\
\text { Muj }\end{array}$ & gl & $\mathbf{t}$ & $\mathbf{p}$ & d \\
\hline ENERO & BD & 306 & 3.64 & 1.26 & 375 & 3.64 & 1.23 & 679 & 0.012 & 0.991 & 0.00 \\
\hline OCT & BD & 208 & 3.36 & 1.26 & 211 & 3.27 & 1.31 & 417 & 0.641 & 0.522 & 0.07 \\
\hline ENERO & IA & 306 & 3.91 & 1.19 & 375 & 3.89 & 1.21 & 679 & 0.186 & 0.853 & 0.02 \\
\hline OCT & IA & 208 & 3.57 & 1.24 & 211 & 3.49 & 1.19 & 417 & 0.662 & 0.508 & 0.06 \\
\hline
\end{tabular}

Nota: $B D=B i g$ Data; IA=Inteligencia Artificial ${ }^{*} \mathrm{p}<0.05 ;{ }^{* *} \mathrm{p}<0.01 ;{ }^{* * *} \mathrm{p}<0.001$

Fuente: elaboración propia

\subsection{Diferencias de género en la percepción de beneficios y riesgos hacia la ciencia de datos, Big Data e Inteligencia Artificial en España}

Con respecto a la segunda pregunta de investigación (PI2), después de analizar la percepción de beneficios y riesgos que los ciudadanos identifican en una serie de aplicaciones asociadas tanto al Big Data como a la Inteligencia Artificial, se ha observado la diferencia que presentan por géneros. En el caso del Big Data las aplicaciones analizadas son: tomar decisiones basadas en datos, conocer el mercado y a los consumidores, hacer predicciones basadas en datos, usar grandes volúmenes de datos, la ciberseguridad, proteger los datos personales, usar redes sociales y mejorar los sistemas de transporte y movilidad. En el caso de la Inteligencia Artificial, por otra parte, las aplicaciones analizadas son: automatizar el trabajo, detectar fraude online, la selec- 
ción de personal, armamento y defensa, la aplicación en medicina para detectar enfermedades, la prevención de catástrofes y la gestión de emergencias en tiempo real.

En la primera oleada de enero, en Big Data, podemos observar que hay varias aplicaciones con diferencias significativas por género en los beneficios percibidos. El uso de grandes volúmenes de datos es más beneficioso para hombres que para mujeres, también lo es tomar decisiones basadas en datos, mejorar los sistemas de transporte y movilidad, hacer predicciones basadas en datos y la Ciberseguridad. En el resto de aplicaciones aunque las diferencias no son significativas, se observa que los hombres presentan mayores beneficios percibidos que las mujeres en todos los casos (Tabla 8).

En cuanto a los riesgos del Big Data de la primera oleada de enero, las diferencias entre hombres y mujeres tampoco son significativas e igualmente se pude observar que los hombres perciben más riesgos que las mujeres en casi todas las aplicaciones salvo en el caso del uso de grandes volúmenes de datos aunque las diferencias son mucho más leves que en los beneficios. Vemos que el mayor tamaño de efecto en los riesgos estaría en la protección de los datos personales (Tabla 8).

Tabla 8. Percepciones por género de beneficios y riesgos de las principales aplicaciones del Big Data en la primera oleada de Enero 2020

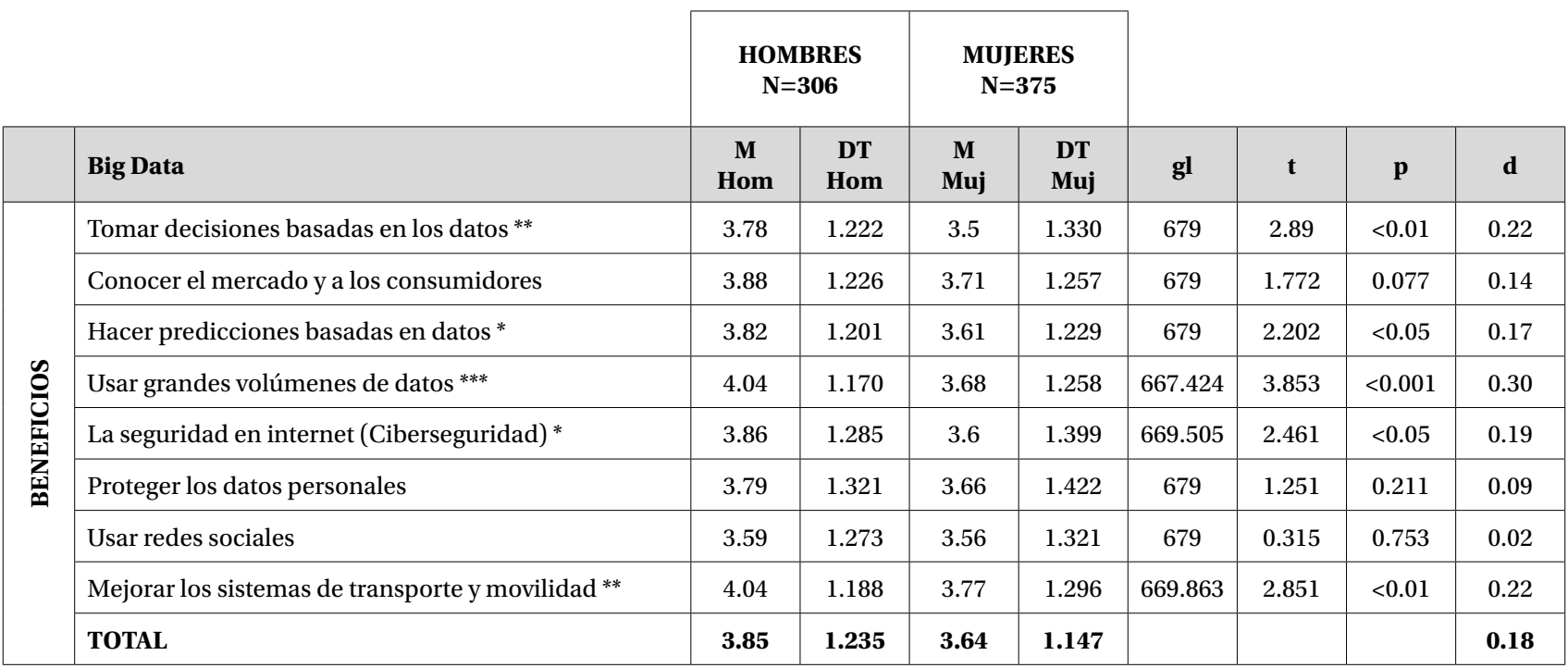




\begin{tabular}{|c|c|c|c|c|c|c|c|c|c|}
\hline \multirow{9}{*}{ ঠ્ટ } & Tomar decisiones basadas en los datos & 3.25 & 1.364 & 3.17 & 1.408 & 679 & 0.702 & 0.483 & 0.06 \\
\hline & Conocer el mercado y a los consumidores & 3.18 & 1.423 & 3.1 & 1.417 & 679 & 0.771 & 0.441 & 0.06 \\
\hline & Hacer predicciones basadas en datos & 3.31 & 1.349 & 3.26 & 1.312 & 679 & 0.474 & 0.636 & 0.04 \\
\hline & Usar grandes volúmenes de datos & 3.23 & 1.443 & 3.25 & 1.390 & 679 & -0.231 & 0.817 & 0.01 \\
\hline & La seguridad en internet (Ciberseguridad) & 3.53 & 1.374 & 3.47 & 1.458 & 679 & 0.554 & 0.58 & 0.04 \\
\hline & Proteger los datos personales & 3.58 & 1.394 & 3.41 & 1.416 & 679 & 1.567 & 0.117 & 0.12 \\
\hline & Usar redes sociales & 3.5 & 1.380 & 3.41 & 1.405 & 679 & 0.832 & 0.406 & 0.06 \\
\hline & Mejorar los sistemas de transporte y movilidad & 3.15 & 1.457 & 3.06 & 1.516 & 679 & 0.776 & 0.438 & 0.06 \\
\hline & TOTAL & $\mathbf{3 . 3 4}$ & 1.398 & 3.27 & 1.241 & & & & 0.05 \\
\hline
\end{tabular}

En la primera oleada de enero, en cuanto a Inteligencia Artificial solamente se aprecian dos diferencias significativas por género en los beneficios percibidos. En el caso de la automatización del trabajo los hombres perciben más beneficios que las mujeres y en armamento y defensa también los hombres perciben significativamente más beneficios que las mujeres.

En el resto de aplicaciones podemos ver que los beneficios percibidos por los hombres son superiores en general a los que perciben las mujeres (Tabla 9).

En cuanto a los riesgos de la Inteligencia Artificial no hay diferencias significativas entre hombres y mujeres, pero sí se aprecia que en algunas aplicaciones los riesgos percibidos son más elevados entre las mujeres que entre los hombres, como en el caso de la automatización del trabajo, la prevención de catástrofes y la gestión de emergencias en tiempo real. Esto nos puede llevar a pensar que el nivel de desconfianza en mujeres sería superior que en hombres. El tamaño de efecto superior se puede observar en la aplicación de nuevos métodos de selección de personal, con mayor riesgo percibido en hombres (Tabla 9). 
Tabla 9. Percepciones por género de beneficios y riesgos de las principales aplicaciones de la Inteligencia Artificial en la primera oleada de Enero 2020

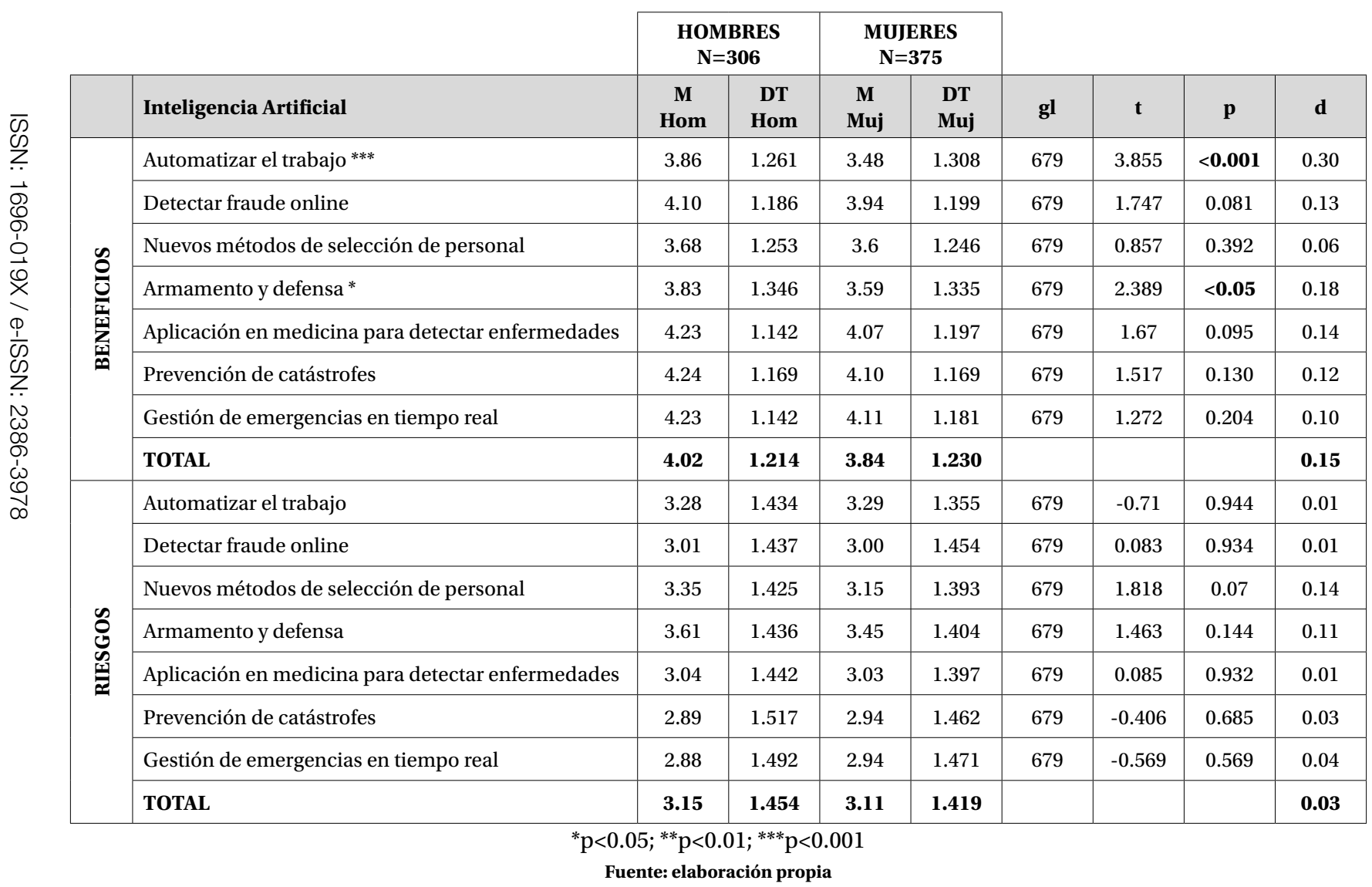

Analizando la segunda oleada de octubre 2020, en cuanto a los beneficios percibidos en el Big Data se observa que las diferencias entre hombres y mujeres no son significativas en ningún caso, pero que los hombres siguen percibiendo mayores beneficios que las mujeres, aunque la distancia se ha reducido entre ellos y las mujeres suben su percepción superando a los hombres en algunas aplicaciones.

Así, en la segunda oleada, las mujeres perciben más beneficios que los hombres en la protección de los datos personales, conocer el mercado y a los consumidores, usar grandes volúmenes de datos, la Ciberseguridad y el uso de redes sociales (Tabla 10). 
La media total de beneficios de Big Data en la primera oleada era de 3.85 en hombres y 3.64 en mujeres (d=0.18), mientras que en la segunda oleada la media es de 3.75 en hombres y 3.74 en mujeres $(\mathrm{d}=0.01)$, con lo que se aprecia esa subida en la percepción de beneficios en mujeres que equilibra las diferencias de género (Tabla 9 y Tabla 10).

Revisando los riesgos percibidos del Big Data en la segunda oleada observamos que solamente una diferencia es significativa, en el caso del uso de grandes volúmenes de datos, que las mujeres perciben mayores riesgos que los hombres con un tamaño de efecto más salto que el resto de casos. Sin embargo se puede ver claramente que ha subido la percepción de riesgos en todas las aplicaciones en las mujeres, superando a los hombres. En la primera oleada solamente había una percepción de mayor riesgo en mujeres en la automatización del trabajo, la prevención de catástrofes y la gestión de emergencias en tiempo real. Sin embargo, en la segunda oleada se eleva esa percepción de riesgos y a pesar de que los hombres también han elevado los riesgos percibidos, las mujeres superan a los hombres en todos los casos (Tabla 10).

La media de riesgos percibidos de Big Data en la primera oleada era en hombres de 3.34 y en mujeres de 3.27 (d=0.05) y en la segunda oleada es en hombres de 3.59 y en mujeres de $3.71(\mathrm{~d}=0.09)$, con lo que se ve claramente la subida y el aumento de la diferencia (Tabla 9 y Tabla 10).

Tabla 10. Percepciones por género de beneficios y riesgos hacia las principales aplicaciones del Big Data en la segunda oleada Octubre 2020

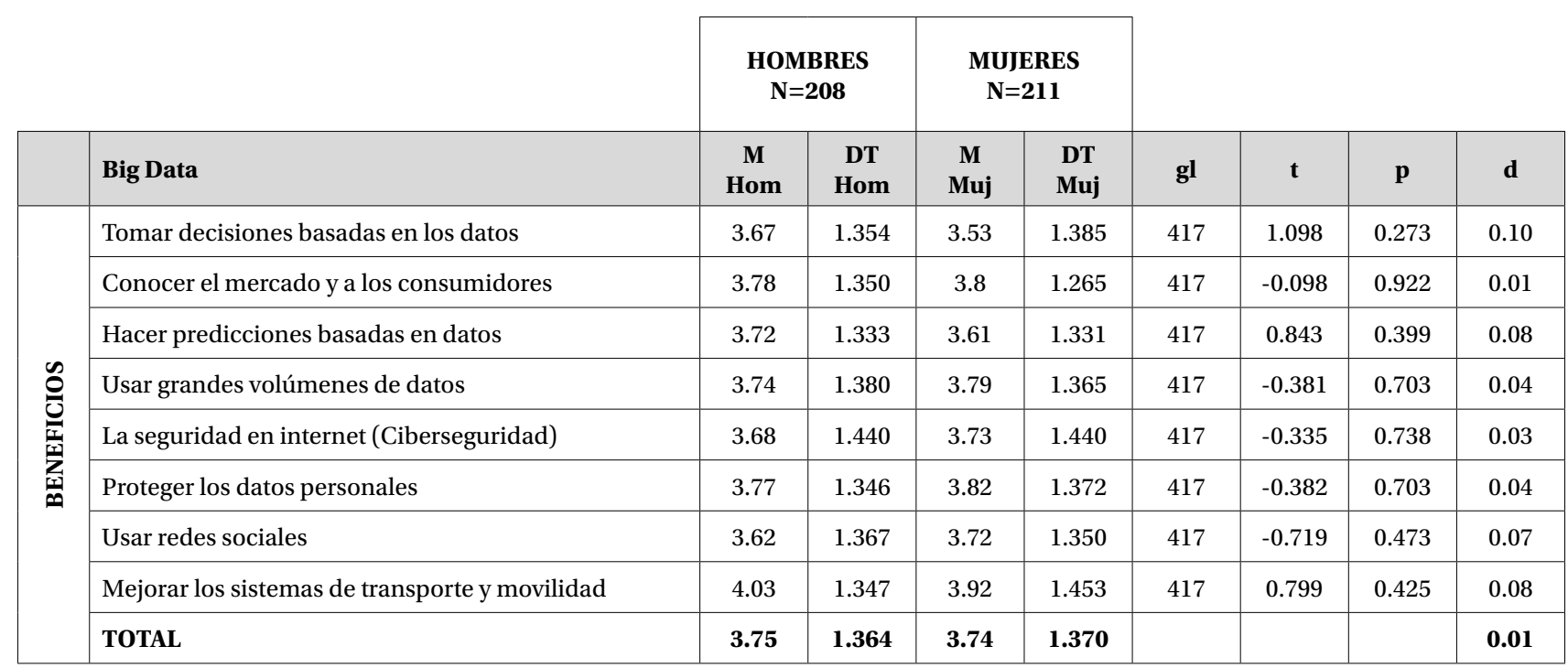




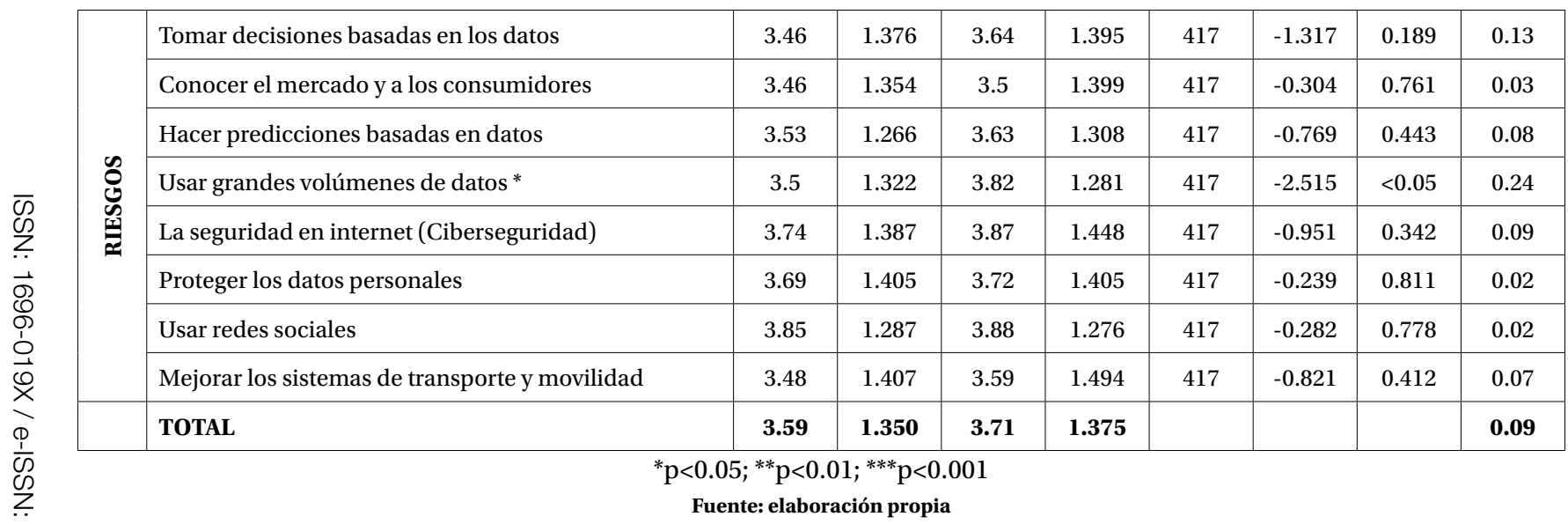

En cuanto a la Inteligencia Artificial, en la segunda oleada de octubre, los beneficios percibidos bajan en hombres y se mantienen en mujeres, pero ello hace que las mujeres superen a los hombres.

La única aplicación en que los hombres superan a las mujeres en beneficios percibidos es en la automatización del trabajo, además es la que tiene un tamaño de efecto mayor que el resto de aplicaciones. En cuanto a tamaño de efectos los más grandes en el caso de las mujeres que perciben más beneficios que los hombres son la aplicación en medicina para detectar enfermedades y la gestión de emergencias en tiempo real (Tabla 11).

La media de beneficios percibidos de la Inteligencia Artificial en la primera oleada era en hombres de 4.02 y en mujeres de 3.84 $(\mathrm{d}=0.15)$, mientras que en la segunda oleada es de 3.77 en hombres y 3.79 en mujeres $(\mathrm{d}=0.01)$ con lo que se aprecia el descenso en ambos casos y que ahora las mujeres superan a los hombres en beneficios percibidos (Tabla $10 \mathrm{y}$ Tabla 11).

En cuanto a los riesgos percibidos de la Inteligencia Artificial en la segunda oleada, tampoco hay datos significativos en diferencias de género. Los riesgos percibidos han descendido ligeramente en hombres, con lo que están un poco más equilibrados.

Los tamaños de efecto mayores los encontramos en detectar fraude online, que los hombres perciben más riesgos que las mujeres y lo mismo sucede en los nuevos métodos de selección de personal.

Solamente hay dos aplicaciones en las que las mujeres perciben más riesgo que los hombres, la prevención de catástrofes y la gestión de emergencias en tiempo real (Tabla 11).

La media de riesgos percibidos de la Inteligencia Artificial en la primera oleada era en hombres de 3.15 y en mujeres de 3.11 $(d=0.03)$, mientras que en la segunda oleada es de 3.48 en hombres y 3.43 en mujeres $(d=0.03)$ con lo que se aprecia que ha subido la percepción de riesgos en ambos casos, aunque los hombres siguen superando a las mujeres pero se acercan más que en el caso del Big Data y se equilibran las diferencias (Tabla 10 y Tabla 11). 
Tabla 11. Percepciones por género de beneficios y riesgos hacia las principales aplicaciones de la Inteligencia Artificial en la segunda oleada Octubre 2020

\begin{tabular}{|c|c|c|c|c|c|c|c|c|c|}
\hline & & $\begin{array}{l}\text { HON } \\
\mathrm{N}=\end{array}$ & $\begin{array}{l}\text { RES } \\
08\end{array}$ & & $\begin{array}{l}\text { RES } \\
11\end{array}$ & & & & \\
\hline & Inteligencia Artificial & $\begin{array}{c}\text { M } \\
\text { Hom }\end{array}$ & $\begin{array}{c}\text { DT } \\
\text { Hom }\end{array}$ & $\begin{array}{c}\text { M } \\
\text { Muj }\end{array}$ & $\begin{array}{l}\text { DT } \\
\text { Muj }\end{array}$ & gl & $\mathbf{t}$ & $\mathbf{p}$ & d \\
\hline & Automatizar el trabajo & 3.62 & 1.403 & 3.40 & 1.432 & 417 & 1.534 & 0.126 & 0.15 \\
\hline & Detectar fraude online & 3.86 & 1.322 & 3.87 & 1.298 & 417 & -0.127 & 0.899 & 0.01 \\
\hline & Nuevos métodos de selección de personal & 3.52 & 1.315 & 3.61 & 1.288 & 417 & -0.687 & 0.493 & 0.01 \\
\hline 응 & Armamento y defensa & 3.45 & 1.347 & 3.47 & 1.487 & 417 & -0.159 & 0.874 & 0.01 \\
\hline$\sum_{x=1}^{\frac{\pi}{1}}$ & $\begin{array}{l}\text { Aplicación en medicina para detectar } \\
\text { enfermedades }\end{array}$ & 3.96 & 1.285 & 4.06 & 1.295 & 417 & -0.794 & 0.428 & 0.08 \\
\hline & Prevención de catástrofes & 4.01 & 1.300 & 4.06 & 1.265 & 417 & -0.414 & 0.678 & 0.04 \\
\hline & Gestión de emergencias en tiempo real & 4.00 & 1.287 & 4.10 & 1.264 & 417 & -0.798 & 0.425 & 0.08 \\
\hline & TOTAL & 3.77 & 1.322 & 3.79 & 1.332 & & & & 0.01 \\
\hline & Automatizar el trabajo & 3.51 & 1.383 & 3.51 & 1.435 & 417 & 0.019 & 0.985 & 0.00 \\
\hline & Detectar fraude online & 3.47 & 1.410 & 3.27 & 1.466 & 417 & 1.430 & 0.154 & 0.14 \\
\hline & Nuevos métodos de selección de personal & 3.62 & 1.310 & 3.47 & 1.381 & 417 & 1.112 & 0.267 & 0.11 \\
\hline$\check{0}$ & Armamento y defensa & 3.85 & 1.276 & 3.76 & 1.422 & 417 & 0.665 & 0.506 & 0.07 \\
\hline 空 & $\begin{array}{l}\text { Aplicación en medicina para detectar } \\
\text { enfermedades }\end{array}$ & 3.45 & 1.375 & 3.4 & 1.405 & 417 & 0.326 & 0.745 & 0.03 \\
\hline & Prevención de catástrofes & 3.25 & 1.406 & 3.29 & 1.489 & 417 & -0.276 & 0.783 & 0.03 \\
\hline & Gestión de emergencias en tiempo real & 3.2 & 1.420 & 3.31 & 1.526 & 417 & -0.737 & 0.462 & 0.07 \\
\hline & TOTAL & 3.48 & 1.368 & 3.43 & 1.446 & & & & 0.03 \\
\hline
\end{tabular}

Respondiendo a la PI2a, se puede observar que los beneficios percibidos descienden y aumentan los riesgos en la segunda olea$\mathrm{da}$, con lo que las diferencias se modifican y en algunos casos pasan a ser negativas, y así los riesgos superan a los beneficios. Las mujeres dejan ver un aumento mayor de los riesgos percibidos, mientras que los hombres no descienden tanto la percepción de beneficios, pero sí se aprecia en casos de aplicaciones concretas no siempre coincidentes con las mujeres.

En el Big Data, la primera oleada muestra una media total de beneficios en hombres de 3.85 (DT=1.235) y en mujeres de 3.64 $(\mathrm{DT}=1.147)(\mathrm{d}=0.18)$, mientras que la media total de riesgos en hombres es de $3.34(\mathrm{DT}=1.398)$ y en mujeres de $3.27(\mathrm{DT}=1.241)$ ( $(=0.05)$ (Tabla 8). Tanto en percepción de riesgos como de beneficios los hombres superan a las mujeres. 
En la segunda oleada la media total de beneficios en hombres es de $3.75(\mathrm{DT}=1.364)$ y en mujeres de 3.74 (DT=1.370) (d=0.01) y la media total de riesgos en hombres es de 3.59 (DT=1.350) y en mujeres de 3.71 (DT=1.375) (d=0.09) (Tabla 10). La percepción de beneficios ha subido en mujeres hasta casi equilibrar con los hombres, mientras que los riesgos percibidos en mujeres han subido hasta superar a los hombres.

En la Inteligencia Artificial, la primera oleada muestra una media total de beneficios en hombres de 4.02 (DT=1.214) y en mujeres de $3.84(\mathrm{DT}=1.230)(\mathrm{d}=0.15)$, mientras que la media total de riesgos en hombres es de 3.15 (DT=1.454) y en mujeres de 3.11 $(\mathrm{DT}=1.419)(\mathrm{d}=0.03)$ (Tabla 9). Los hombres igualmente presentan más beneficios que riesgos pero superan a las mujeres en ambas en la primera oleada.

En la segunda oleada, la media total de beneficios en hombres es de 3.77 (DT=1.322) y en mujeres de 3.79 (DT=1.332) ( $d=0.01$ ), mientras que la media total de riesgos en hombres es de 3.48 (DT=1.368) y en mujeres de 3.43 (DT=1.446) (d=0.03) (Tabla 11). Descienden los beneficios percibidos en hombres y también en mujeres, pero éstas superan a los hombres en los beneficios, no así en riesgos a pesar de que aumentan.

\section{Discusión y conclusiones}

La sociedad actual está acostumbrada a escuchar y leer términos como Big Data e Inteligencia Artificial por su uso (y abuso) en los medios de comunicación en los últimos años. Sin embargo, el hecho de que se hayan convertido en habituales en el lenguaje no significa que la mayoría de las personas sepa en realidad todos los matices y los aspectos que conllevan. El periodismo científico ha ido ganando espacio no solo en la prensa especializada sino también en la generalista y la "guerra" por los datos no ha hecho más que reafirmar el poder que tienen y el de aquellos que saben manejarlos e interpretarlos. El uso de los datos permite a los medios de comunicación ofrecer mejores contenidos informativos, especialmente en el periodismos de investigación, y su incorporación de manera atractiva hace que el público valore de manera más positiva la información al hacer más atractiva la información transmitida. Una reivindicación que ha ido ganando peso en los últimos años es el hecho de asociar la ciencia con lo masculino siendo un debate, el de género, muy en boga en la actualidad.

La incorporación de estas nuevas aplicaciones tecnológicas tiene implicaciones en cuanto a los procesos de trabajo, pero también en cuanto a los conocimientos base que los ciudadanos necesitan adquirir para manejarlos con seguridad y para sentirse confiados. Este artículo analiza el conocimiento que la sociedad tiene de la ciencia de los datos, en concreto, de la comprensión del Big Data y de la Inteligencia Artificial, pero además, también se ha medido su relación con el género. De esto modo, las preguntas de investigación planteadas pretendían medir si había diferencias de género en el grado de conocimiento e interés y en la percepción de beneficios y riesgos de la ciencia de datos, el Big Data o la Inteligencia Artificial. Además, se midió si el confinamiento que se produjo en la sociedad española en el año 2020, entre marzo y junio, por Covid-19 tuvo algún impacto en las respuestas de los encuestados.

La muestra analizada mostró un alto grado de familiaridad con los conceptos de ciencia de datos, Big Data e Inteligencia Artificial, siendo mayor este último. La Inteligencia Artificial tuvo un mayor interés para los hombres que para las mujeres mientras que en los otros dos conceptos, Ciencia de Datos y Big Data, el interés fue similar en ambos grupos. La pandemia y el boom informativo que supuso, basado, principalmente, en informaciones repletas de datos sin apenas tiempo para el análisis de los mismo, 
además, no consiguió que cambiara, en gran medida el interés de los encuestados sobre estos términos, pero sí se pudo observar que disminuyó ligeramente.

Sobre la percepción de beneficios y riesgos, tanto los hombres como las mujeres perciben más beneficios que riesgos en la ciencia de datos, el Big Data y la Inteligencia Artificial, aunque son los hombres quienes muestran puntuaciones más altas en cuanto a los beneficios, sin embargo las mujeres mostraron, en general, una percepción de riesgos más elevada. En cuanto a esta pregunta, el confinamiento por Covid-19 sí que influyó en las respuestas ya que, por ejemplo, en el caso de las aplicaciones de Big Data, la muestra general percibía más beneficios que riesgos en la mayoría de las aplicaciones. En la comparativa por género, en el caso de las mujeres percibieron más riesgos que beneficios en casi todas las aplicaciones, mientras que los hombres seguían percibiendo más beneficios que riesgos.

Se observa que en cuanto a diferencias de género no hay resultados significativos, pero sí líneas que apuntan a lo que se observa en estudios previos, porque las mujeres presentan una percepción de los riesgos más elevada que los hombres quienes mostraron un mayor interés en la Inteligencia Artificial por lo que queda aún lejos separar ciencia del género masculino, aunque se aprecia una tendencia a la baja en esto.

Esta encuesta parece contradecir la idea de Bustamante Alonso y Guillén Alonso (2017) quienes indicaban que la sociedad está inmersa en una era disruptiva y las tecnologías avanzan más rápido que la capacidad de adaptación social pues según las respuestas recogidas los entrevistados conocen y valoran de forma positiva la ciencia de datos, la Inteligencia Artificial y el Big Data. En este sentido, parece que ante el miedo que estos conceptos podían generar hace años en la sociedad, han ido ganando peso las ventajas que tienen frente a los posibles inconvenientes, gracias a la normalización y a la incorporación no solo de estos conceptos sino de lo que transmiten los medios de comunicación, en los que cada vez es más habitual acompañar la información con visualizaciones de datos. Además, el hecho de que los datos de organismos oficiales deban ser publicados y accesibles a la población genera una mayor confianza en la utilidad que estos tienen al fortalecer la transparencia de las instituciones en aras de una mayor confiabilidad.

Contrastando los resultados con la reciente encuesta de Percepción Social de la Ciencia y la Tecnología podemos observar que se aprecia una tendencia similar. Los hombres perciben en general más beneficios que las mujeres en la Inteligencia Artificial, salvo en la franja más joven, entre 15 y 24 años, en la que los hombres elevan su percepción de riesgo por encima de las mujeres de la misma edad. Las mujeres perciben más riesgos que los hombres en las franjas de edad entre los $25 \mathrm{y}$ los 44 especialmente y en la de mayores de 65 años. En cuanto a la robotización del trabajo que es la otra variable que se puede comparar del estudio de la Fecyt, los hombres perciben más riesgos que las mujeres en la franja entre los 15 a 34 años, que coincide con la finalización de estudios y la etapa de incorporación al mercado laboral. En este aspecto las mujeres no se muestran convencidas, porque la mayoría de respuestas se sitúan en un punto intermedio, quizá porque sería preciso incorporar más información a los ciudadanos para alimentar su capacidad crítica de valorar el uso de estas tecnologías en su entorno laboral sin sentirse amenazados (FECYT, 2021).

No se debe obviar el poder que tiene la ciencia de datos, la Inteligencia Artificial y el Big Data para resolver algunos de los mayores problemas de la humanidad en el contexto actual, en la denominada Cuarta Revolución Industrial en la que los datos y el dominio que de ellos se tenga resultará fundamental. Esta revolución ha propiciado, además, el surgimiento de nuevas profesiones 
ligadas a este sector como el director de datos, el ingeniero de datos, el científico de datos, el director de seguridad de la información o el delegado de protección de datos, entre otros.

Los datos, los grandes números, se han integrado a la vida diaria no solo a nivel informativo sino también, de manera significativa, al mercado pues las empresas han entendido que son vitales a la hora de generar negocio. La ciencia de datos ha supuesto, además, la creación de puestos de trabajo ya que la economía basada en los datos estimulará la investigación y la innovación, aportando más oportunidades de negocio y aumentando la disponibilidad de conocimiento y capital, en particular para las pequeñas y medianas empresas (PYME), en toda Europa (Monleón-Getino, 2015). Quizás esta pueda ser la razón fundamental por la que los encuestados perciben más beneficios que riesgos en la ciencia de datos, la Inteligencia Artificial y el Big Data. Además, resulta también significativo que las respuestas de los encuestados no presenten grandes diferencias entre hombres y mujeres por lo que se puede hablar de que cada vez es menor la distancia de género en este campo.

En este sentido, convendría realizar en el futuro otro estudio de corte similar que permita realizar una investigación de corte longitudinal para poder constatar si estos resultados son hechos aislados o si vaticinan un cambio de tendencia en el campo de estudio. Así mismo, sería interesante completar este estudio con la combinación de diferentes metodologías, como entrevistas en profundidad que permitan ahondar en los datos con respuestas que puedan explicar el porqué de los resultados obtenidos. El tema tiene la suficiente importancia y relevancia en la sociedad actual, como demuestra que el número de profesionales en ciencia de datos se ha duplicado en los últimos cuatro años (LinkedIn, 2015), para profundizar más en él, por lo que sería recomendable realizar una encuesta con una muestra mayor en el futuro.

\section{Agradecimientos}

Este artículo ha sido traducido al inglés por Charles E. Arthur.

\section{Referencias bibliográficas}

Bauer, M. W., y Jensen, P. (2011). The mobilization of scientists for public engagement. Public Understanding of Science, 20(1), 3-11. https://doi.org/10.1177/0963662510394457

Berman, F. D. y Bourne, P. E. (2015). Let's Make Gender Diversity in Data Science a Priority Right from the Start. PLOS Biology, 13 (7). https://doi.org/10.1371/journal.pbio.1002206

Bustamante Alonso, N. B., y Guillén Alonso, S. Th. (2017). Un acercamiento al Big Data y su uti-lización en comunicación. Mediaciones Sociales, (16), 115-134. https://doi.org/10.5209/MESO.58112

Calvo-Rubio, L. M., Ufarte-Ruiz, Ma J. (2020). Percepción de docentes universitarios, estudiantes, responsables de innovación y periodistas sobre el uso de inteligencia artificial en periodismo. El profesional de la información, v. 29, n. 1, e290109. https://doi. org/10.3145/epi.2020.ene.09

Comisión Interamericana de Mujeres. (2020). COVID-19 en la vida de las mujeres. In OAS Cataloging-in-Publication Data. http:// www.oas.org/cim\%0Ahttp://www.oas.org/es/cim/docs/ArgumentarioCOVID19-ES.pdf 
D’Ignazio, C. y Klein, L. F. (2020). Data feminism. MIT Press.

Díaz Martínez, C., Díaz García, P. y Navarro Sustaeta, P. (2020). Sesgos de género ocultos en los macrodatos y revelados mediante redes neurales: ¿hombre es a mujer como trabajo es a madre?. Revista Española de Investigaciones Sociológicas, 172: 41-60. http://dx.doi.org/10.5477/cis/reis.172.41

European Commission (2014). Special Eurobarometer 419: Public perceptions of science, research and innovation (Issue October). European Commission. https://doi.org/10.2777/95599

European Commission (2017a). Special Eurobarometer 460: Attitudes towards the impact of digitisation and automation on daily life. https://doi.org/10.2759/835661

European Commission (2017b). Special Eurobarometer 464a: Europeans' attitudes towards cyber security Fieldwork (Issue June). European Commission. https://doi.org/10.2838/009088

European Commission (2020). On Artificial Intelligence - A European approach to excellence and trust. https://doi.org/10.1017/ CBO9781107415324.004

FECYT Fundación Española para la Ciencia y la Tecnología (2018). IX Encuesta de Percepción Social de la Ciencia y la Tecnología 2018. https://icono.fecyt.es/sites/default/files/filepublicaciones/18/epscyt2018_informe_0.pdf

FECYT Fundación Española para la Ciencia y la Tecnología. (2021). Percepción social de la ciencia y la tecnología en España 2020. https://icono.fecyt.es/sites/default/files/filepublicaciones/21/percepcion_social_de_la_ciencia_y_la_tecnologia_2020_ informe_completo_0.pdf

Felt, U. (ed). (2007). Optimising public understanding of science and technology, 610-644.

Finucane, M. L., Slovic, P., Mertz, C. K., Flynn, J. y Satterfield, Th. A. (2000). Gender, race, and perceived risk: The "white male" effect. Health, Risk \& Society, 2(2), 159-172. https://doi.org/10.1080/713670162

Hayes, B. C., y Tariq, V. N. (2000). Gender differences in scientific knowledge and attitudes toward science: a comparative study of four Anglo-American nations. Public Understanding of Science, 9(4), 433-447. https://doi.org/10.1088/0963-6625/9/4/306

Howard, A., Borenstein, J. (2018). The Ugly Truth About Ourselves and Our Robot Creations: The Problem of Bias and Social Inequity. Sci Eng Ethics, 24, 1521-1536. https://doi.org/10.1007/s11948-017-9975-2

Instituto de las Mujeres y para la Igualdad de Oportunidades. (2020). "La perspectiva de género, esencial en la respuesta a la COVID-19.” In Catálogo de publicaciones de la Administración General del Estado.

Jurgenson, N. (2014). View from nowhere. The New Inquiry. October 9. https://thenewinquiry.com/essays/view-from-nowhere/ Leavy, S. (2018). "Gender bias in artificial intelligence: the need for diversity and gender theory in machine learning". In Proceedings of the 1st International Workshop on Gender Equality in Software Engineering (GE '18). Association for Computing Machinery, New York, NY, USA, 14-16. https://doi.org/10.1145/3195570.3195580

Mayer-Schönberger, V., \& Cukier, K. (2013). Big Data. La revolución de los datos masivos. Turner Publicaciones S.L.

McQuillan, D. (2018). Data science as machinic neoplatonism. Philosophy \& Technology, 31(2), 253-272. https://doi.org/10.1007/ s13347-017-0273-3 
Miller, J. D. (2004). Public Understanding of, and Attitudes toward, Scientific Research: What We Know and What We Need to Know. Public Understanding of Science, 13(3), 273-294. https://doi.org/10.1177/0963662504044908

Miller, S. (2001). Public understanding of science at the crossroads. Public Understanding of Science, 10(1), 115-120. https://doi. org/10.3109/a036859

Monleón Getino, A. (2015). El impacto del Big-data en la Sociedad de la Información. Significado y utilidad. Historia y Comunicación Social, 20(2), 427-445. https://doi.org/10.5209/rev_HICS.2015.v20.n2.51392

Montaña Blasco, M., Ollé Castellà, C., y Lavilla Raso, M. (2020). Impacto de la pandemia de Covid-19 en el consumo de medios en España. Revista Latina, (78), 155-167. https://doi.org/10.4185/RLCS-2020-1472

ONU Mujeres. (2021). Los efectos del COVID-19 sobre las mujeres y las niñas. UnWomen. https://interactive.unwomen.org/ multimedia/explainer/covid19/es/index.html

Palomares Ruiz, A. (2004). Profesorado y educación para la diversidad en el siglo XXI. Universidad de Castilla la Mancha.

Pearson, G. (2001). The participation of scientists in public understanding of science activities: The policy and practice of the U.K. Research Councils. https://doi.org/10.3109/a036860

Samoili, S., López Cobo, M., Gómez, E., De Prato, G., Martínez-Plumed, F., \& Delipetrev, B. (2020). AI Watch - Defining Artificial Intelligence. Towards an operational definition and taxonomy of artificial intelligence. In Joint Research Centre (European Commission). https://doi.org/10.2760/382730

Sánchez-Holgado, P., Arcila-Calderón, C., y Frías-Vázquez, M. (2021). El papel de los y las periodistas españoles ante la comunicación de la ciencia de datos en medios en línea. Revista Prisma Social, (32), 344-375. https://revistaprismasocial.es/article/ view/3901

Tannenbaum, C., Ellis, Robert P., Eyssel, F., Zou, J. y Schiebinger, L. (2019). Sex and gender analysis improves science and engineering. Nature, 575, 137-146. https://doi.org/10.1038/s41586-019-1657-6 